International Journal of Pure and Applied Mathematics

Volume $97 \quad$ No. $3 \quad$ 2014, 353-357

ISSN: 1311-8080 (printed version); ISSN: 1314-3395 (on-line version)

url: http://www.ijpam.eu

doi: http://dx.doi.org/10.12732/ijpam.v97i3.7

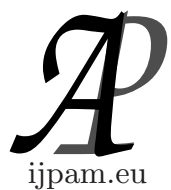

\title{
THERMAL STAGE OF GROWTH OF "TWO PHASE" BUBBLE CONTAINING HEATED PARTICLE
}

\author{
Nail S. Khabeev ${ }^{1}$, Mona AL-Mannai ${ }^{2}$ \\ ${ }^{1,2}$ University of Bahrain \\ P.O. Box 32038, KINGDOM OF BAHRAIN
}

\begin{abstract}
The thermal regime of vapor bubble growth around spherical solid heated particle in a liquid is studied. Such problems arise at different kind of accidents, followed by a vapor explosion.
\end{abstract}

AMS Subject Classification: 00A69

Key Words: heated particle, vapor bubble, thermal regime

\section{Introduction}

The study of large scale vapor explosions is a very complex task. The difficulties here are related to the influence on the process of a large number of factors in the complex. Because of the big number of defining parameters capabilities of the physical modeling are limited. So, the creation of mathematical models of the process reflecting its characteristic features is actual. This opens up opportunities for creation of scientific methods for predicting the consequences of accidents causing vapor explosions. Review of papers devoted to this problem, is given in [1].

In the present paper we consider a single aspect of the problem at the microlevel. A formula is derived that describe the variation of the thickness of the vapor layer in time.

Received: July 9, 2014

(C) 2014 Academic Publications, Ltd.

$\S_{\text {Correspondence author }}$ url: www.acadpubl.eu 
In a similar statement the dynamics of a vapor layer around a spherical particle in a shock wave was studied numerically in [2].

\section{The Formulation of the Problem and Basic Equations}

Suppose that a hot particle get into the cold liquid. This can occur, for example, in case of accidents at nuclear power plants, when melted nuclear fuel get into the cooling system of a nuclear reactor. We introduce a spherical coordinate system centered at the center of the particle. We assume that the process of heat and mass transfer around the particle is spherically symmetric.During contact of hot particle with cold liquid a film boiling begins on the surface of the particle. This process accompanied by the growth of the vapor shell around the particle [3].

Consider the asymptotic stage of vapor shell growth when in and around the shell is established steady-state distribution of temperature $[2[$. Such a temperature distribution around the solid particle heated to temperature $T_{0}>$ $T_{s}(p)$ has the form

$$
\begin{gathered}
T_{v}=\left(T_{0}-T_{s}\right) \frac{r_{0}}{R-r_{0}}\left(\frac{R}{r}-1\right)+T_{s}, \quad r_{0}<r<R \\
T_{l}=\left(T_{s}-T_{\infty}\right) \frac{R}{r}+T_{\infty}, r>R
\end{gathered}
$$

Here $T$ is temperature. The subscripts $\nu$ and $l$ attributed to the parameters of vapor and liquid respectively. Indices $s$ and $\infty$ classified accordingly to the parameters on the saturated line and far away from the particle. $R$ is the radius of the vapor shell, $r_{0}$ is the radius of the particle, $r-$ spherical Euler coordinate ,$p$ is the pressure.

The problem under consideration belongs to the class of Stefan type since it includes a moving boundary at which the phase transition takes a place.

We write the boundary conditions on the surface of the vapor shell [3],[4]

$$
r=R(t): \quad \lambda_{l} \frac{\partial T_{1}}{\partial r}-\lambda_{v} \frac{\partial T_{v}}{\lambda r}=j l, \quad T_{v}=T_{l}=T_{s}
$$

Here $\lambda$ is the coefficient of thermal conductivity, $j$ is the rate of phase transition from a unit of surface, $l$ is the latent heat of evaporation.

If, for simplicity, we assume that the growth of the shell is determined by the evaporation of the liquid only, the rate of phase transitions can be associated 
with the rate of growth of the shell's radius:

$$
j=\rho_{v} \frac{d R}{d t}
$$

where $\rho_{v}$ is the vapor density.

Calculating the heat fluxes at the interface from (1) the boundary condition (2) using (3) can be written in the form:

$$
-\lambda_{l} \frac{T_{s}-T_{\infty}}{R}+\lambda_{v} \frac{\left(T_{0}-T_{s}\right) r_{0}}{\left(R-r_{0}\right) R}=p_{v} l \frac{d R}{d t}
$$

It is shown in [2] that at significant intervals of time the temperature of the heated particle is changing very slowly due to its high thermal capacity.

On the asymptotic stage of growth of vapor shell inertial effects are not significant . So, the pressure in the system is also changing slowly. Therefore, for simplicity, we can take:

$$
T_{0}=\text { const }, \quad T_{s}(p)=\text { const }
$$

Let us assume, for simplicity, that the thermophysical parameters of the phases are also constants. Then equation (4) is an ordinary differential equation of the first order with respect to the radius of the shell. we rewrite it as:

$$
\begin{gathered}
\frac{d R}{d t}=\frac{\alpha}{R}+\frac{\beta}{R\left(R-r_{0}\right)} \\
\alpha=-\frac{\lambda_{l}\left(T_{s}-T_{\infty}\right)}{l \rho_{v}}, \quad \beta=\frac{\lambda_{v}\left(T_{0}-T_{s}\right) r_{0}}{l \rho_{v}}
\end{gathered}
$$

Equation (5) is equation with separable variables. Therefore, we rewrite it as:

$$
d t=\frac{R\left(R-r_{0}\right) d R}{\alpha\left(R-r_{0}\right)+\beta}
$$

Integrating equation (6) we obtain the dependence of the radius of the shell from time in implicit form:

$$
\frac{R^{2}-R_{0}^{2}}{2 \alpha}-\frac{\beta}{\alpha^{2}}\left(R-R_{0}\right)-\frac{\beta}{\alpha^{2}} R_{*} \ln \left|\frac{R-R_{*}}{R_{0}-R_{*}}\right|=t, \quad R_{*}=-\frac{\beta}{\alpha}+r_{0}
$$

here $R_{0}$ is the value of the radius of the shell at time $t=0$. Qualitative analysis of solution (7) shows that it has a singularity at $R=R_{*}$. Solution tends to this value asymptotically. 
If the initial radius of the vapor shell $R_{0}$ is less than $R_{*}$, then the vapor shell radius is increasing to this critical value $R_{*}$. If, on the contrary, the initial radius of the shell is greater than $R_{*}$, then the vapor condenses in the shell and its radius decreases to the value $R_{*}$.

The physical explanation for this behavior is the following:

If $R=R_{*}$ the heat fluxes from the vapor and liquid phases to the interphase surface are balanced. Thus

$$
r=R: \quad \lambda_{l} \frac{\partial T_{1}}{\partial r}=\lambda_{v} \frac{\partial T_{v}}{\partial r}
$$

Therefore according to the boundary condition (2) $j=0:\left(\frac{d R}{d t}=0\right)$. So, the equilibrium condition is implemented.

The value of the critical radius $R_{*}$ can be rewritten as

$$
R_{*}=r_{0}\left[1+\frac{\lambda_{\nu}}{\lambda_{l}} \frac{\left(T_{0}-T_{s}\right)}{\left(T_{s}-T_{\infty}\right)}\right]
$$

The fact that the solution tends asymptotically to this state is demonstrating the stability of this state.

Review of the recent publications on the dynamics of bubbles is given in [6].

\section{Conclusion}

The process of growth of a vapor shell around the heated particle in thermal quasi-stationary regime was studied. A formula describing the variation of the radius of the vapor shell in time is obtained. It is shown that under assumption of the constancy of the temperature of the heated particle the radius of the vapor shell asymptotically approaches to a certain equilibrium value.

\section{References}

[1] S.G. Bankoff, Vapor Explosions, A critical Review, In: Proc.of the 6th Int. Heat Transfer Conf. vol 6. Nat.Res. Council of canada, Toronto (1978), 355-366.

[2] N.S. Khabeev, O.R. Ganiev, Dynamics of a vapor shell around a heated particle in a liquid, Journal of Appl. Mech. and techn. Phys., 48, No. 4 (2007), 525-533, doi: 10.1007/s10808-007-0066-z. 
[3] O. Dwayer, Heat Transfer in Boiling Metals, Moscow, Mir (1980).

[4] L.I. Sedov, Continnum Mechanics, World Scientific (1990).

[5] R.I. Nigmatulin, Dynamics Of Multiphase Media, Hemisphere, Washington D.C. (1990), doi: TA357.N52513.

[6] W. Lauterborn, et al, Dynamics of bubble oscillations, Rep. Prog. Phys., 73 (2010), doi: 10.1088/0034-4885/73/10/106501. 
